\title{
TEMPERATURE ANISOTROPIES IN A MAGNETOSPHERIC FTE
}

\author{
M. F. Smith and C. J. Owen
}

Laboratory for Extraterrestrial Physics, NASA/Goddard Space Flight Center.

\begin{abstract}
We present AMPTE UKS data from a well-studied magnetospheric flux transfer event, showing detailed ion phasespace distributions for each region of this layered event. We show that the perpendicular temperature anisotropy maximizes at the center of the event. This is inconsistent with recent suggestions that FTE signatures may result from the spacecraft moving into and then out of the magnetosheath via the plasma depletion layer. We present an explanation for the temperature anisotropy structure in terms of a reconnection model.
\end{abstract}

\section{Introduction}

Magnetic reconnection is widely believed to be the dominant mechanism for the transfer of plasma and momentum across the dayside magnetopause. Many observations support this view, e.g., observations of accelerated flows [Paschmann et al., 1979] and momentum and energy balance across the magnetopause [Sonnerup et al., 1981]. In addition to quasi-steady reconnection, a second class of events, Flux Transfer Events (FTEs), were discovered in ISEE data by Russell and Elphic [1978]. It is now widely accepted that these signatures are manifestations of transient reconnection. Statistical studies [e.g. Southwood et al., 1986] show that FTE occurrence is strongly correlated with a southward interplanetary field and thus support this viewpoint.

The structure of magnetospheric FTEs has been studied in some depth by Rijnbeek et al. [1988] and Farrugia et al. [1988]. However, these studies did not include detailed ion distributions within the events. Previously, Thomsen et al. [1987] presented ion and electron distributions observed inside both magnetospheric and magnetosheath FTEs. Unfortunately, the ISEE plasma instrument used in this study could not determine the phase space distribution parallel to $B$ in the magnetosphere. Klumpar et al. [1990] used the AMPTE CCE mass spectrometer to investigate the plasma composition within a magnetospheric FTE. They found that the composition is unique, again supporting the reconnection scenario. These authors also found that $T_{1}>T_{1}$ for the cold ion population within the FTE. However, the two events studied by Klumpar et al. [1990] showed neither the layered structure of the Farrugia et al. [1988] events, nor any dramatic ion flows.

An alternative explanation for at least some FTEs, and the event studied here in particular, has been put forward by Sibeck $[1990,1992]$. Sibeck suggests that these signatures arise from a brief encounter of a spacecraft with the magnetosheath, due to the magnetopause response to a solar wind pressure transient. In this scenario (see Figure 1a) the spacecraft crosses from the magnetosphere, through both the low-latitude boundary layer (LLBL) and the plasma depletion layer (PDL) [Zwan and Wolf, 1976] and into the magnetosheath. As the magnetopause moves back outwards the spacecraft traverses the aforementioned regions in reverse order. This model predicts similar signatures to the reconnection FTE models and thus leads to some ambiguity in the interpretation of data. Recently, Sibeck and Smith [1992] have shown that the flow velocities both within and outside the event studied in detail by Rijnbeek et al. [1988] and Farrugia et al. [1988] are consistent with a wave passing along the magnetopause. However, their results do not provide conclusive evidence that the event is a magnetopause crossing.

\section{Copyright 1992 by the American Geophysical Union.}

Paper number 92GL01618

$0094-8534 / 92 / 92 \mathrm{GL}-01618 \$ 03.00$
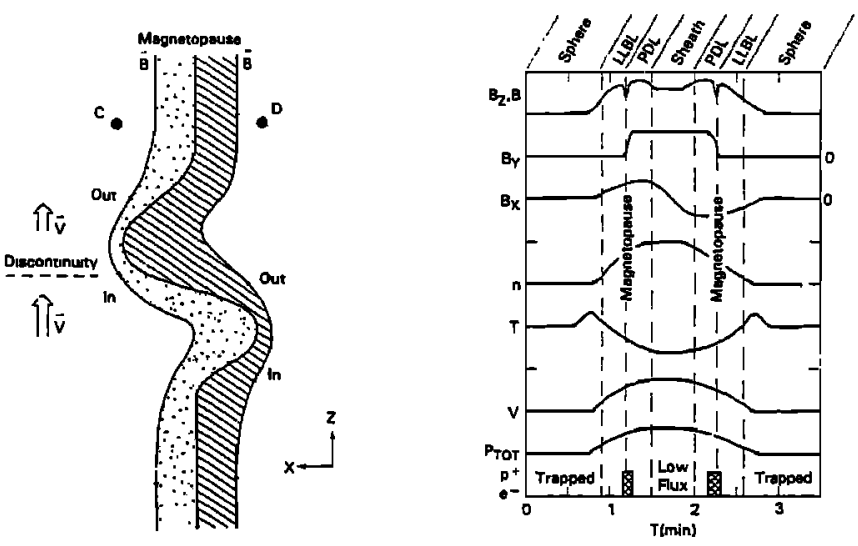

Fig. 1. a) The Sibeck $[1990,1992]$ model in which the spacecraft at D makes a brief crossing of the LLBL, PDL, and magnetosheath due to the passage of a pressure pulse, and $b$ ) the signatures predicted by this model.

Fuselier et al. [1991] have studied the temperature anisotropy within the PDL and found that $T_{1}>T_{1}$, with $T_{1} / T_{1}$ values typically 21.5 . Also, Anderson et al. [1991] found $T_{1} / T_{1}$ values of up to 4 in this region. In each of these studies the anisotropy was greater in the PDL than in the magnetosheath, with the anisotropy increasing as the magnetopause was approached. Crooker and Siscoe [1977], extending the model of Zwan and Wolf [1976], have shown that such an increase is expected within the PDL. These studies suggest that, if the Sibeck [1990,1992] hypothesis is correct, the temperature anisotropy should decrease towards the center of the event as the spacecraft crosses the PDL and then moves into the magnetosheath. We will show that although the region identified by Sibeck as the PDL has a perpendicular temperature anisotropy, the central region has a greater anisotropy. Our observations are thus inconsistent with Sibeck's simple magnetopause crossing picture.

\section{Observations}

In this paper we present high-time resolution ion measurements from the central region of a magnetospheric FTE previously studied by Farrugia et al. [1988]. We use data from the AMPTE UKS ion instrument [Coates et al., 1985] and magnetometer [Southwood et al., 1985]. The former provides a full 3D sample every spacecraft spin ( -5 secs), while the latter has been averaged over the same 5 seconds as the ion data. The UKS ion instrument has the advantage of timeresolution over the CCE plasma instrument at the expense of plasma composition. However, the CCE measurements [Klumpar et al., 1990] show that the majority of the plasma within an FTE are protons ( $>95 \%$ by number density). In addition, Paschmann et al. [1986] have shown that the pressure anisotropies are affected very little by ion species other than protons. Hence we will treat the UKS data as purely protons.

Figure 2 [from Farrugia et al., 1988] shows the magnetic field and plasma parameters within the FTE observed at 1046 UT on 28 October 1984 . The FTE has been split, roughly symmetrically about the center, into four regions. The properties of each region have been described in detail by Farrugia et al. [1988]. 


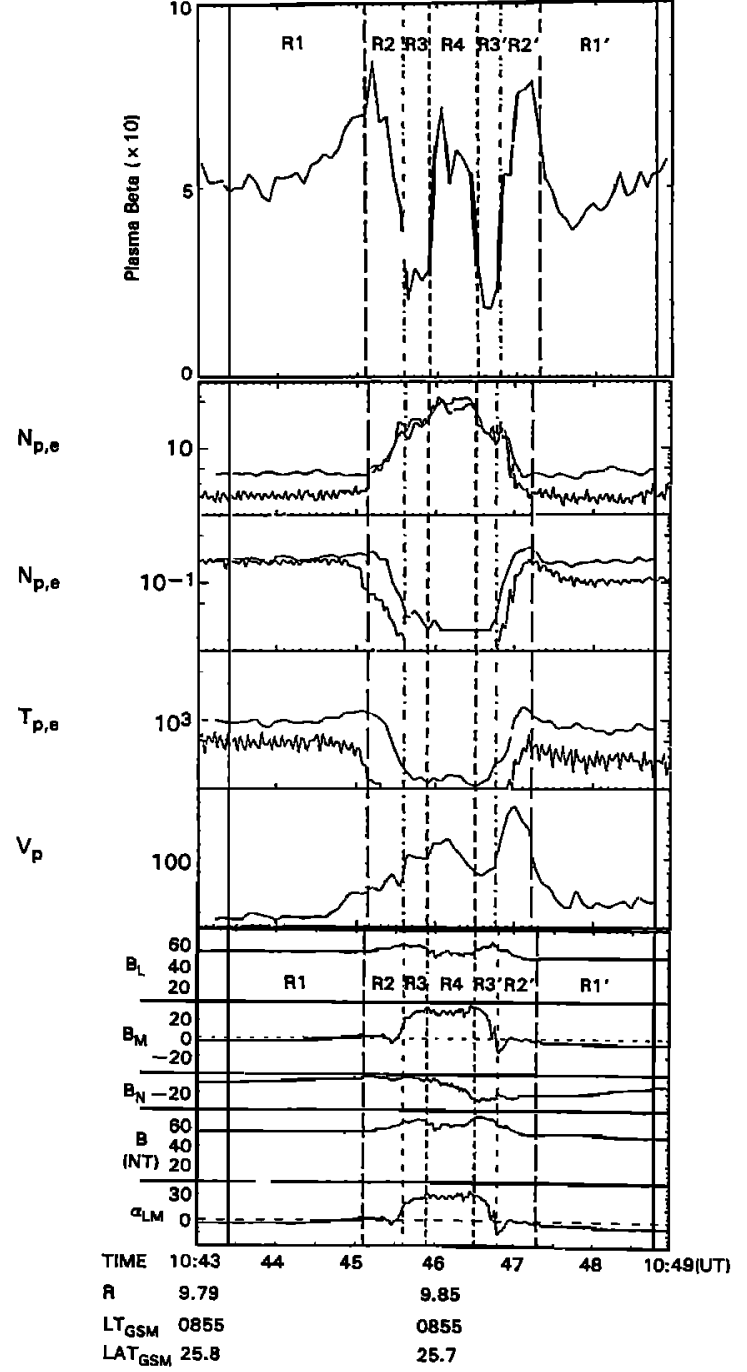

Fig. 2. Data from AMPTE UKS for day 302 of 1984. The panels, from the top, are: Plasma $\beta$; ion (thin line) and electron (thick line) densities in the range $10 \mathrm{eV}-20 \mathrm{keV}$; ion (thin line) and electron (thick line) partial densities for energies $\geq 10$ $\mathrm{keV}$; ion (thin line) and electron (thick line) temperatures; ion flow velocity; 3 components of the B field in boundary normal coordinates; the total field strength; and the B field angle in the LM plane (after Farrugia et al. [1988]). Note the similarities between the signatures and those predicted in Figure $1 \mathrm{~b}$.

Summarizing their conclusions, region 1 is the field line draping region with a magnetospheric plasma population and the field and flow perturbed in accordance with MHD. In region 2 the ion population characteristics are intermediate between those in the magnetosheath and magnetosphere, while the electrons stream bidirectionally along what are thought to be newly reconnected field lines. In region 3 the field strength maximizes, and the plasma is sheath-like, but at a reduced density. Finally, in region 4 the plasma is fully sheath-like in terms of both density and temperature. Note the similarity between these parameters and those predicted by Sibeck [1990] (Figure 1b).

In Figure 3 we show four phase-space density (PSD) plots of the ion data taken at different times within this FTE. We have followed the method of Smith and Rodgers [1991] and compressed the data into 2D by integrating the full 3D distribution onto a plane. The perpendicular velocity is preserved so the resulting PSD is not distorted in any way. The $x$-axis of the plot is parallel to the $5 \mathrm{~s}$ averaged B-field direction, while the $y$ - axis is chosen such that the GSE $\mathrm{x}$-direction is contained within the plane of the plot. Figure 3a $(1045: 20)$ shows the distribution from region 2 on the inbound leg of the spacecraft traversal through the FTE; Figure $3 b$ (1045:46 UT) is from region 3 on the inbound leg; Figure 3c (1046:12 UT) from region 4; and Figure 3d (1046:37 UT) from region 3 on the outbound leg. The contour levels and velocity scales have been chosen to show both magnetospheric and magnetosheath populations in Figure 3a, but only the sheath population (as there is no significant magnetospheric population) in Figures 3b-d.

The PSD plot for region 2 (Figure 3a) shows a virtually isotropic population of both magnetospheric and magnetosheath plasma. By the start of region 3 inbound (Figure $3 \mathrm{~b}$ ) the magnetospheric plasma has disappeared and we see only sheathlike plasma at a reduced density. The plot clearly shows a temperature anisotropy with $T_{1} / T_{1} \approx 1.6$. Figure $3 c$, from the core of the FTE (region 4), again shows a temperature anisotropy, $T_{1} / T_{1} \approx 2.2$, and a higher density than region 3 . Finally, Figure 3d shows a distribution from region 3 on the outbound leg. Again there is a temperature anisotropy with $T_{1} / T_{1} \approx 1.9$.


Fig. 3. Ion phase space distributions at a) 10:45:20 in region 2 (top left); b) 10:45:46 in region 3 inbound (top right); c) 1046:12 in region 4 (bottom left); and d) 10:46:37 in region 3 outbound (bottom right).

In Figure 4, shown as the dashed line, we have taken a cut through the region 2 distribution (1045:20) along the $y=0$ axis, i.e. parallel to $B$. This data is compared in the figure to similar cuts taken in the field line draping region (1045:04, solid line) and on the region 2/region 3 boundary (1045:30, dot-dashed line). As the spacecraft moves from region 1 into region 2 the high energy magnetospheric plasma disappears. At the same time low density sheath plasma appears, first at the upper end of the typical sheath energy range (e.g. 1045:20). By 1045:30, the sheath population is evident across its full energy range albeit at lower densities. In Figure 5b we show the variation of the temperature anisotropy throughout regions 2,3 and 4 of the FTE. Note that region 2 contains a mixture of magnetospheric and magnetosheath plasma resulting in an almost isotropic distribution. However, it is clear that the perpendicular temperature anisotropy is larger in region 4 than in region 3. Investigation of the individual temperature components (Figure 5c) shows that $T_{\perp}$ is relatively constant throughout regions 3 and 4 . However, $T_{1}$ decreases through region 3 inbound and region 4 and then increases across region 3 outbound. The anisotropy peak in region 4 thus results from a reduction in $T_{r}$ 


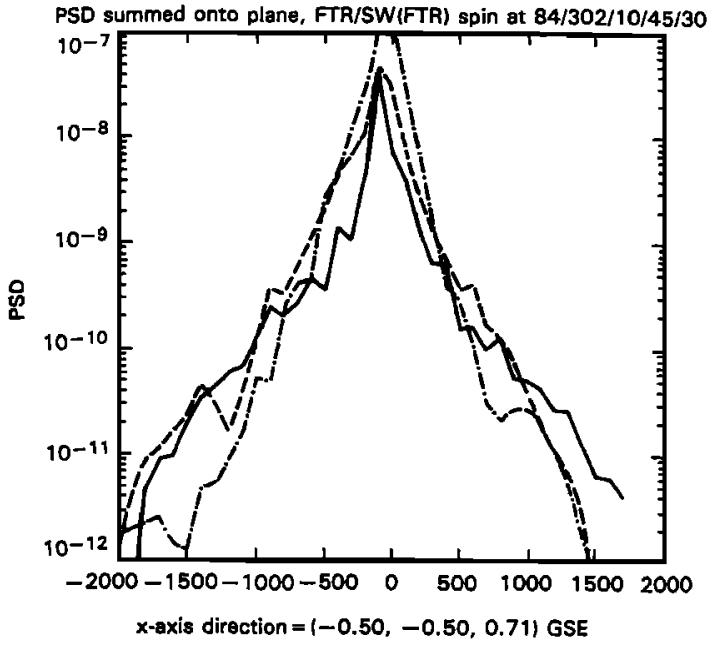

Fig. 4. Cuts through the PSDs along the $\mathrm{y}=0$ axis for 10:45:04 (region 1, solid line), 10:45:20 (region 2, dashed line), and 10:45:30 (region $2 / 3$ boundary, dot-dashed line).

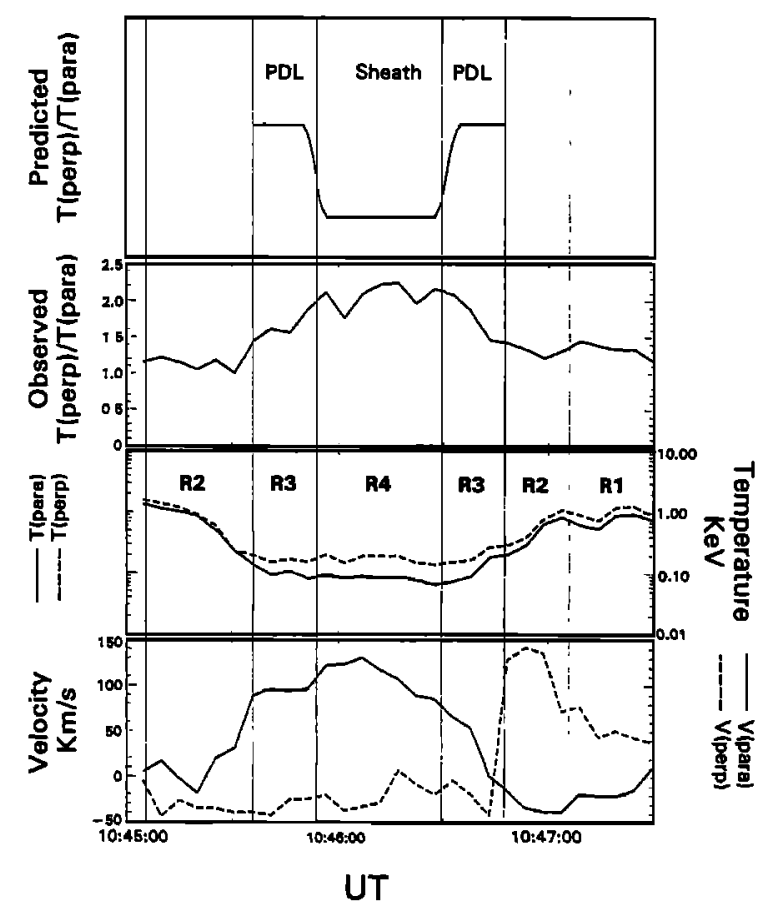

Fig. 5. a) Schematic variation of the temperature anisotropy (linear scale) in regions 3 and 4 predicted by Sibeck [1990, 1992]; b) the measured anisotropy in these regions; c) parallel (solid line) and perpendicular (dashed line) temperatures; and d) parallel (solid line) and perpendicular (dashed line) velocities.

The parallel velocity (Figure 5d) clearly peaks in region 4 at $\sim 130 \mathrm{~km} \mathrm{~s}^{-1}$. The perpendicular velocity is approximately constant, with the exception of a large flow burst in region 2 outbound. This burst was noticed by Farrugia et al. [1988] and has been discussed by Sibeck and Smith [1992].

\section{Discussion}

The temperature anisotropies measured in both regions 3 and 4 of this FTE $\left(T_{1}>T_{1}\right)$ are opposite to those found by Klumpar et al. [1990]. However, their events show no layered structure and had virtually zero flow. As the event studied here is typical of the UKS events studied by Farrugia et al. [1988], we would expect the anisotropy structure shown in Figure 5 to be typical. However, firmly establishing this is difficult due to instrumental considerations: even for this event, one of the longest duration events seen by UKS, the number of unaliased 3D plasma measurements within the various regions is small. Statistical confirmation of the plasma structure must thus wait. However, we can use the above measurements to test whether this observed signature is consistent with transient reconnection and/or a brief crossing into the magnetosheath.

The temperature anisotropy structure required by the Sibeck $[1990,1992]$ pressure pulse model is shown schematically in Figure 5a, where the regions 3 and 4 of the FTE have been identified with the PDL and magnetosheath. As the cone angle of the IMF is less than $45^{\circ}$ at this time [Sibeck, 1992], a perpendicular temperature enhancement in the magnetosheath is expected [Noerdlinger, 1964]. Indeed, both regions 3 and 4 do show a perpendicular anisotropy which would appear to be consistent with these assumptions. However, the measured temperature anisotropy varies in the opposite manner to that required by the Sibeck model as the spacecraft traverses between the two regions. Also, region 3 has no streaming hot ion population leaking out of the magnetosphere as observed in the PDL by AMPTE CCE [Fuselier et al., 1991]. Hence, on the basis of current understanding, region 3 does not correspond to the PDL, and we conclude that this event cannot be interpreted as a simple crossing of the spacecraft into the magnetosheath.

On the basis of the temperature anisotropies alone, this event cannot be clearly identified as reconnection associated, as there are at present no predictions for the temperatures expected inside an FTE. However, the data can be interpreted in terms of motion through a number of layers resulting from reconnection at the dayside magnetopause. Clearly, as pointed out by Farrugia et al. [1988], region 1 is the draping region. We identify region 2 as the electron boundary layer, where the field lines are newly opened. On entry to region 2 we first see the loss of magnetospheric ions coming from the X-line direction, and then the loss of those coming from the ionosphere as the flux tube empties of high-energy plasma. The only sheath particles seen at first are streaming electrons, which have mirrored in the ionosphere and thus show a bidirectional flow.

Further into region 2 some high energy magnetosheath ions are seen. These ions are likely to constitute the edge of the ion boundary layer [Gosling et al., 1990]. Curiously, these sheathlike ions have virtually no bulk flow along the field line. These are unlikely to be diffusing from region 3 (older reconnected field lines with more of a sheath population at lower velocities) as the width of region 2, based on the perpendicular velocities in the center of the event, is a few hundred ion gyroradii. The region $2 /$ region 3 boundary is signified by a large shear in the magnetic field which we associate with an Alfven wave propagating from the $\mathrm{X}$-line. Thus, the sheath ions in region 2 must have had speeds exceeding the Alfven speed and may have been partially scattered by this wave.

After the field shear we see, in region 3, a D-shaped magnetosheath-like ion distribution characteristic of reconnection. The flattened shape of the distribution in the parallel directions comes from two effects: i) the sheath itself has a perpendicular temperature anisotropy and ii) only those ions with a parallel velocity greater than the de Hoffman-Teller velocity can get through the magnetopause. This second effect causes the distribution to be D-shaped [Cowley, 1982; Smith and Rodgers, 1991], and results in an increase in the parallel bulk flow velocity. Region 4 again has a D-shaped distribution although the parallel flow velocity is larger in this region. This may be due to older reconnected field lines being picked up by an increasing sheath flow as they move away from the reconnection site (the ion flow speed depending on both the sheath Alfven speed and flow speed [e.g. Cowley and Owen, 1989]). Thus, we identify both regions 3 and 4 as reconnection layers 
with region 3 being more newly-opened. We can combine the above scenario with the explanation of the ion flows by Sibeck and Smith [1992]. These authors show that the flows are consistent with a wave, but they do not attempt to explain the flow data in terms of either a MP crossing or a reconnection picture. Having excluded the former model, we suggest that there is an initial inward boundary motion, possibly from a pressure pulse, which causes the spacecraft to pass through firstly newly reconnected field lines and then older ones. A rapid outward motion then results in high speed flows in a sunward direction in region 2 outbound (see Figure 2), and moves the magnetopause well away from the UKS location. Note this picture places no requirements on the nature of the reconnection, i.e. whether it is quasi-steady or transient. Any changes in the reconnection rate will cause the thickness of the reconnection layers to vary with time. These temporal effects may be superposed on the spatial effects described above.

\section{Summary}

Ion data from a crossing of a magnetospheric event, previously explained as either an FTE or a magnetopause crossing, shows an increasing perpendicular temperature anisotropy towards the center of the event. This is inconsistent with the simple magnetopause crossing model. However, the data may be explained as a transient encounter, caused by wave-like boundary motion, with a series reconnection layers. In this scenario there is no crossing of the magnetopause itself.

Acknowledgments. We thank A.J. Coates and D.J. Southwood for the ion and magnetometer data respectively, R.A. Gowen, M.A. Birdseye and E. Mazur for programming support, and the referees for their comments. CJO was supported by a NRCGSFC Research Associateship.

\section{References}

Anderson, B.J., S.A. Fuselier, and D. Murr, Electromagnetic ion cyclotron waves observed in the plasma depletion layer, Geophys. Res. Lett., 18, 1955, 1991.

Coates, A.J., et al., AMPTE UKS 3-dimensional ion experiment, IEEE Trans. Geosci. Remote Sens., GE-23, 287, 1985.

Cowley, S.W.H., The causes of convection in the Earth's magnetosphere: A review of developments during the IMS, Rev. Geophys. Space Phys., 20, 531, 1982.

Cowley, S.W.H., and C.J. Owen, A simple illustrative model of open flux tube motion over the dayside magnetopause, Planet. Space Sci., 37, 1461, 1989.

Crooker, N.U., T.E. Eastman, and G.S. Stiles, Observations of plasma depletion in the magnetosheath at the dayside magnetopause, J. Geophys. Res., 84, 869, 1979.

Crooker, N.U., and G.L. Siscoe, A mechanism for pressure anisotropy and mirror instability in the dayside magnetosheath, J. Geophys, Res., 82, 185, 1977.
Farrugia, C.J., et al., A Multi-Instrument study of flux transfer event structure, J. Geophys. Res., 93, 14465, 1988.

Fuselier, S.A., et al., $\mathrm{He}^{2+}$ and $\mathrm{H}^{+}$dynamics in the subsolar magnetosheath and plasma depletion layer, J. Geophys. Res., $96,21095,1991$.

Gosling, J.T., et al., The electron edge of the low latitude boundary layer during accelerated flow events, Geophys. Res. Lett., 17, 1833, 1990.

Klumpar, D.M., S.A. Fuselier, and E.G. Shelley, Ion composition measurements within magnetospheric flux transfer events, Geophys. Res. Lett., 17, 2305, 1990.

Noerdlinger, P.D., Wave generation near the outer boundary of the magnetosphere, J. Geophys. Res., 69, 369, 1964.

Paschmann, G., et al., Plasma acceleration at the Earth's magnetopause: Evidence for reconnection, Nature, 282, 243, 1979.

Paschmann, G., et al., The magnetopause for large magnetic shear: AMPTE/IRM observations., J. Geophys. Res., 9I, $11099,1986$.

Rijnbeek, R.P., et al., A Magnetic boundary signature within flux transfer events, Planet. Space. Sci., 35, 871, 1988.

Russell, C.T., and R.C. Elphic, Initial ISEE magnetometer results: Magnetopause observations, Space Sci. Rev., 22, 681, 1978.

Sibeck, D.G., A model for the transient magnetospheric response to sudden solar wind dynamic pressure variations, $J$. Geophys. Res., 95, 3755, 1990.

Sibeck, D.G., Transient events in the outer magnetosphere: boundary waves or FTEs?, J. Geophys. Res., accepted, 1992.

Sibeck, D.G., and M.F. Smith, Magnetospheric plasma flows associated with boundary waves and flux transfer events, Geophys. Res. Lett., accepted, 1992.

Smith, M.F., and D.J. Rodgers, Ion distributions at the dayside magnetopause, J. Geophys. Res., 96, 11617, 1991.

Sonnerup, B.U.O., et al., Evidence for magnetic field reconnection at the Earth's magnetopause, J. Geophys. Res., 86, 10049,1981 .

Southwood, DJ., W.A.C. Mier-Jedrzejowicz, and C.T. Russell, The fluxgate magnetometer for the AMPTE UK subsatellite, IEEE Trans. Geosci. Remote Sens., GE-23, 301, 1985.

Southwood, D.J., et al., A survey of flux transfer events recorded by the UKS spacecraft magnetometer, Planet. Space Sci., 34, 1349, 1986.

Thomsen, M.F., et al., Ion electron velocity distributions within flux transfer events, J. Geophys. Res., 92, 12127, 1987.

Zwan, B.J., and R.A. Wolf, Depletion of solar wind plasma near a planetary boundary, J. Geophys. Res., 81, 1636, 1976.

C.J. Owen and M.F. Smith, Code 696, NASA/GSFC, Greenbelt, MD 20771, USA.

(Received: March 10, 1992;

Revised June 11, 1992;

Accepted: June 30, 1992) 